Case Report

\title{
Squamous Cell Carcinoma of the Lung with Metastasis to the GI Tract Associated with EGFR Exon 19 Deletion
}

\author{
Glen J. Weiss, ${ }^{1,2,3}$ Agnes K. Liman, ${ }^{4,5}$ Jeffrey Allen, $^{6}$ \\ Philip Y. Cheung, ${ }^{2}$ and Rajesh N. Kukunoor ${ }^{7}$ \\ ${ }^{1}$ Cancer Treatment Centers of America, Goodyear, AZ 85338, USA \\ ${ }^{2}$ The Translational Genomics Research Institute, Phoenix, AZ 85004, USA \\ ${ }^{3}$ CRAB Clinical Trials Consortium, Seattle, WA 98101, USA \\ ${ }^{4}$ Veterans Affairs Pittsburgh Healthcare System, Pittsburgh, PA 15206, USA \\ ${ }^{5}$ University of Pittsburgh School of Medicine, Pittsburgh, PA 15261, USA \\ ${ }^{6}$ Humboldt Medical Specialists, Eureka, CA 95501, USA \\ ${ }^{7}$ Ironwood Cancer and Research Centers, Phoenix, AZ 85028, USA \\ Correspondence should be addressed to Glen J. Weiss; glen.weiss@ctca-hope.com
}

Received 23 April 2013; Accepted 29 August 2013

Academic Editor: Gottfried J. Locker

Copyright (C) 2013 Glen J. Weiss et al. This is an open access article distributed under the Creative Commons Attribution License, which permits unrestricted use, distribution, and reproduction in any medium, provided the original work is properly cited.

We describe three confirmed cases of squamous cell carcinoma (SCC) of the lung with metastasis to the gastrointestinal (GI) tract, with two having epidermal growth factor receptor (EGFR) exon 19 deletions in all available specimens. One of these patients received EGFR tyrosine kinase directed therapy for a brief period with some symptom relief. Consideration of EGFR exon 19 mutation testing in SCC of the lung, particularly for those with GI tract metastasis, may identify this potentially drug-targetable entity.

\section{Introduction}

Squamous cell carcinoma (SCC) of the lung metastasizing to the GI tract is an uncommon occurrence [1-4]. Epidermal growth factor receptor (EGFR) mutations have been identified in approximately $9 \%$ of metastatic SCC of the lung in one series [5]. We report on three patients with metastatic SCC of the lung metastasizing to the gastrointestinal (GI) tract, two of whom had tumor with a confirmed EGFR exon 19 deletion.

\section{Case Presentations}

Case 1. A 39-year-old never-smoker man presented with cough, headaches, night sweats, 7-pound weight loss, and constipation for several weeks and was found to have multifocal metastases involving the lung, brain, and colon. A diagnostic colonoscopy was performed, and pathology confirmed metastatic SCC. Additional samples from the lung obtained by bronchoscopy demonstrated SCC consistent with a primary nonsmall cell lung cancer (NSCLC). He then underwent craniotomy and resection for a solitary cerebellar metastasis with pathology consistent with metastatic SCC of the lung. Subsequently, he was treated with cisplatin and gemcitabine, followed by vinorelbine and docetaxel for up to 3 cycles before developing extracranial disease progression. Tissues from the lung, brain, and colon underwent independent expert pathology review and confirmed metastatic SCC of the lung. Because of his never-smoker status, the brain metastasis sample was sent for EGFR mutation testing (exons 18-21) and found to have an EGFR exon 19 deletion. The patient was started on erlotinib and had a transient clinical response with resolution of night sweats and 4-pound weight gain. Three months later, progression by radiographic evidence of bone metastases was observed, and he passed away three weeks later. No postprogression sample was available for analysis.

Case 2. A 79-year-old man with a 20-pack-year smoking history who was diagnosed with a $6.2 \mathrm{~cm}$ stage IIA T2bNOM0 SCC lung cancer underwent surgical resection. He also had 
TABLE 1: Analysis of tumor samples.

\begin{tabular}{lcc}
\hline & Location of tumor sample & Presence of EGFR exon 19 deletion (Y/N) \\
\hline Case 1 & Lung, cerebellar, mediastinal lymph nodes from stations 2, 7, 10R, and 4R, and colon & Y in all 7 samples \\
Case 2 & Lung and colon & Y in both samples \\
Case 3 & Sigmoid colon & $\mathrm{N}$ \\
\hline
\end{tabular}

a remote history of transitional cell carcinoma of the bladder. Adjuvant chemotherapy was not offered. Nearly 10 months later a colon metastasis was detected, and biopsy of the tumor was consistent with SCC of the lung. He subsequently developed brain metastasis and died approximately 28 months after his NSCLC diagnosis. Both the lung primary and colon metastasis were tested for and confirmed the presence of an EGFR exon 19 deletion.

Case 3. A 60-year-old man with an 80-pack-year smoking history and a periampullary cancer status after Whipple resection approximately 17 years earlier presented with decreased caliber of his bowel movements. A colonoscopy revealed a nearly obstructing distal colonic mass. While undergoing further workup, a lung mass was identified and biopsied confirming SCC of the lung. Immunohistochemical (IHC) staining was positive for CK5/6 and p63 and negative for CA19-9 and TTF-1. A laparoscopic segmental resection confirmed metastatic SCC of the lung. IHC staining of this specimen was positive for $\mathrm{CK} 5 / 6, \mathrm{CK} 7$, and $\mathrm{p} 63$ and negative for CK20 and CDX-2. Unfortunately, the patient had a prolonged hospital course with respiratory complications and development of abscesses and died approximately 6 weeks later. Available tumor from the sigmoid colon was tested for an EGFR exon 19 deletion, and an exon 19 deletion was not identified.

Table 1 outlines the available tumor samples from all three cases that were assessed for EGFR exon 19 deletion.

\section{Discussion}

Of the three confirmed SCC of the lung with metastasis to the GI tract, two had EGFR exon 19 deletions. We did not detect any discordance for the mutation findings, as both primary and metastatic tumor from the first two cases had the EGFR exon 19 deletions. This suggests that EGFR exon 19 deletions are present in the initial primary tumor clone that has metastatic potential. While Case 3 did not have a detected EGFR exon 19 deletion, it is unlikely that if we had available primary tumor tissue, this deletion would be detected. Because of limitations of tumor tissue sample for Cases 2 and 3, evaluation of EGFR mutation status was focused on exon 19 deletion.

One of two patients (Case 1) received EGFR tyrosine kinase directed therapy for a brief period with some symptom relief. One can only speculate that if the EGFR exon 19 mutation was identified earlier in his disease course, there may have been improved clinical benefit.

\section{Conclusion}

Consideration of EGFR exon 19 mutation testing in SCC of the lung, particularly for those with GI tract metastasis, may identify this potentially drug-targetable entity.

\section{Consent}

All tumor tissues analyzed in this study were retrospectively obtained after prior approval of the Translational Genomics Research Institute under Exemption 4 of Title 45 Code of Federal Regulations (CFR) concerning retrospective study of existing data. Patient consent is not required under this exemption and was not obtained for this study, as Title 45 CFR Part 46 does not apply. The clinical information associated with these specimens is not individually identifiable and was collected in such a manner that subjects cannot be identified either directly or indirectly through identifiers linked to the subjects.

\section{Conflict of Interests}

Glen J. Weiss is on the speaker's bureau for Genentech and Pfizer. He has received speaker's fees from Medscape, Quintiles, Eli Lilly, Cephalon, Merrimack, and Roche/Ventana. The other authors have no conflict of interests to declare.

\section{References}

[1] D. Carroll and P. B. Rajesh, "Colonic metastases from primary squamous cell carcinoma of the lung," European Journal of Cardiothoracic Surgery, vol. 19, no. 5, pp. 719-720, 2001.

[2] C.-J. Yang, J.-J. Hwang, W.-Y. Kang et al., "Gastro-intestinal metastasis of primary lung carcinoma: clinical presentations and outcome," Lung Cancer, vol. 54, no. 3, pp. 319-323, 2006.

[3] T. E. Stinchcombe, M. A. Socinski, L. M. Gangarosa, and A. H. Khandani, "Lung cancer presenting with a solitary colon metastasis detected on positron emission tomography scan," Journal of Clinical Oncology, vol. 24, no. 30, pp. 4939-4940, 2006.

[4] S. Y. Kim, H. K. Ha, S. W. Park et al., "Gastrointestinal metastasis from primary lung cancer: CT findings and clinicopathologic features," American Journal of Roentgenology, vol. 193, pp. W197-W201, 2009.

[5] J. Cadranel, A. Mauguen, M. Faller et al., "Impact of systematic EGFR and KRAS mutation evaluation on progressionfree survival and overall survival in patients with advanced non-small-cell lung cancer treated by erlotinib in a French prospective cohort (ERMETIC project-part 2)," Journal of Thoracic Oncology, pp. 71490-71502, 2012. 


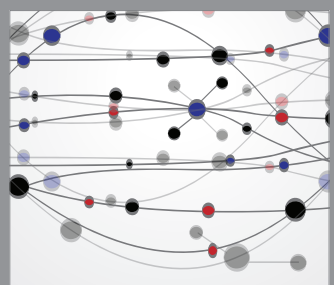

The Scientific World Journal
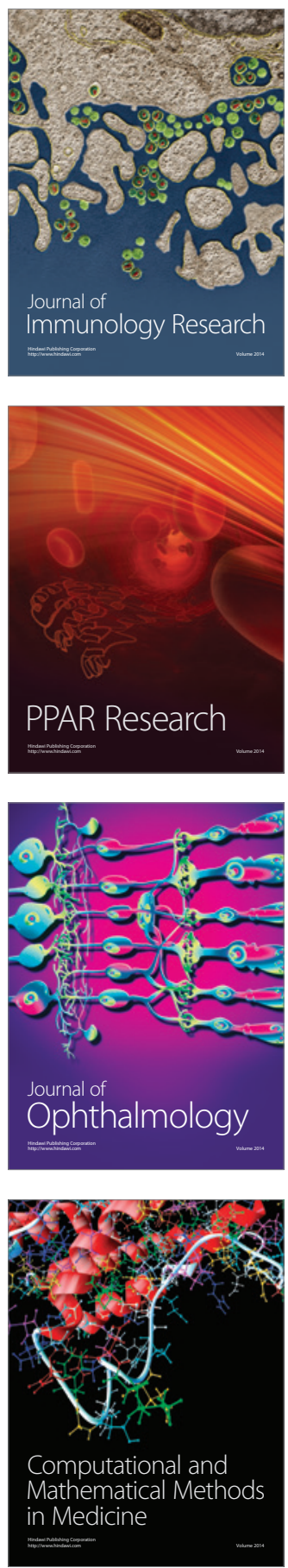

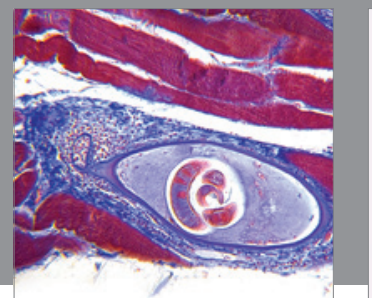

Gastroenterology

Research and Practice
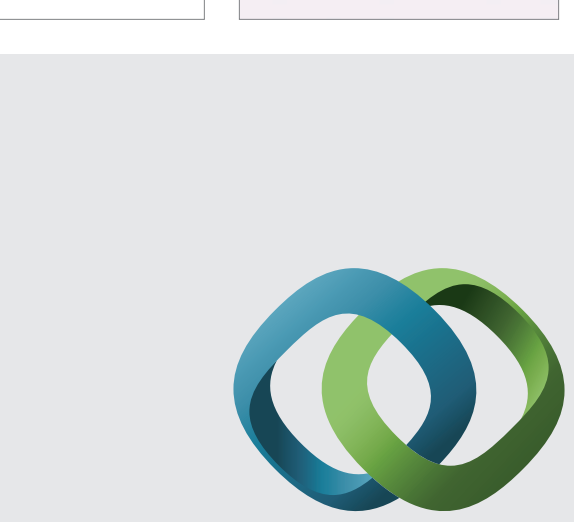

\section{Hindawi}

Submit your manuscripts at

http://www.hindawi.com
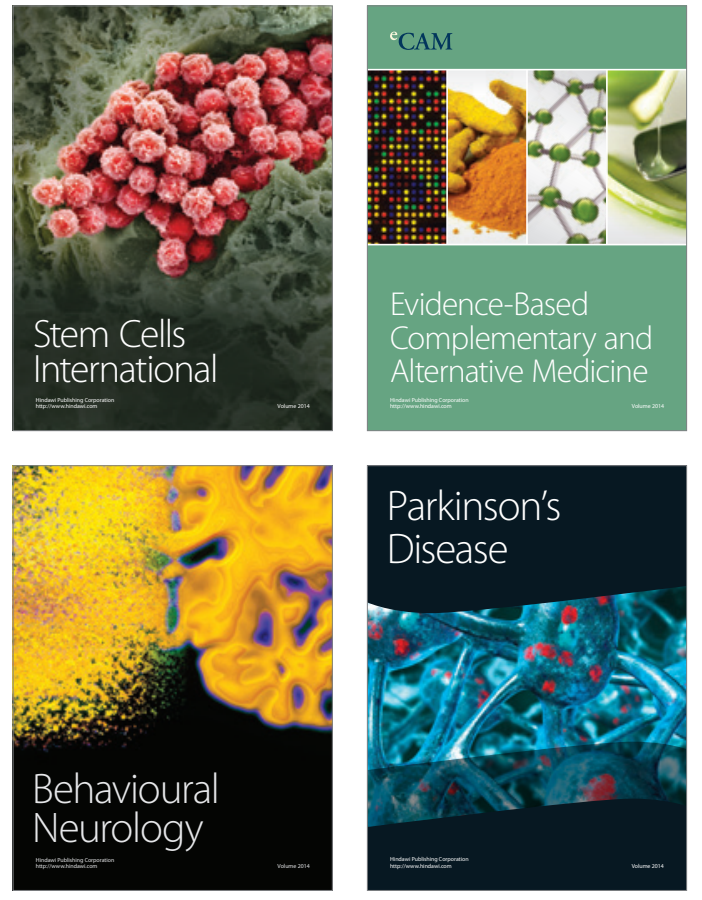
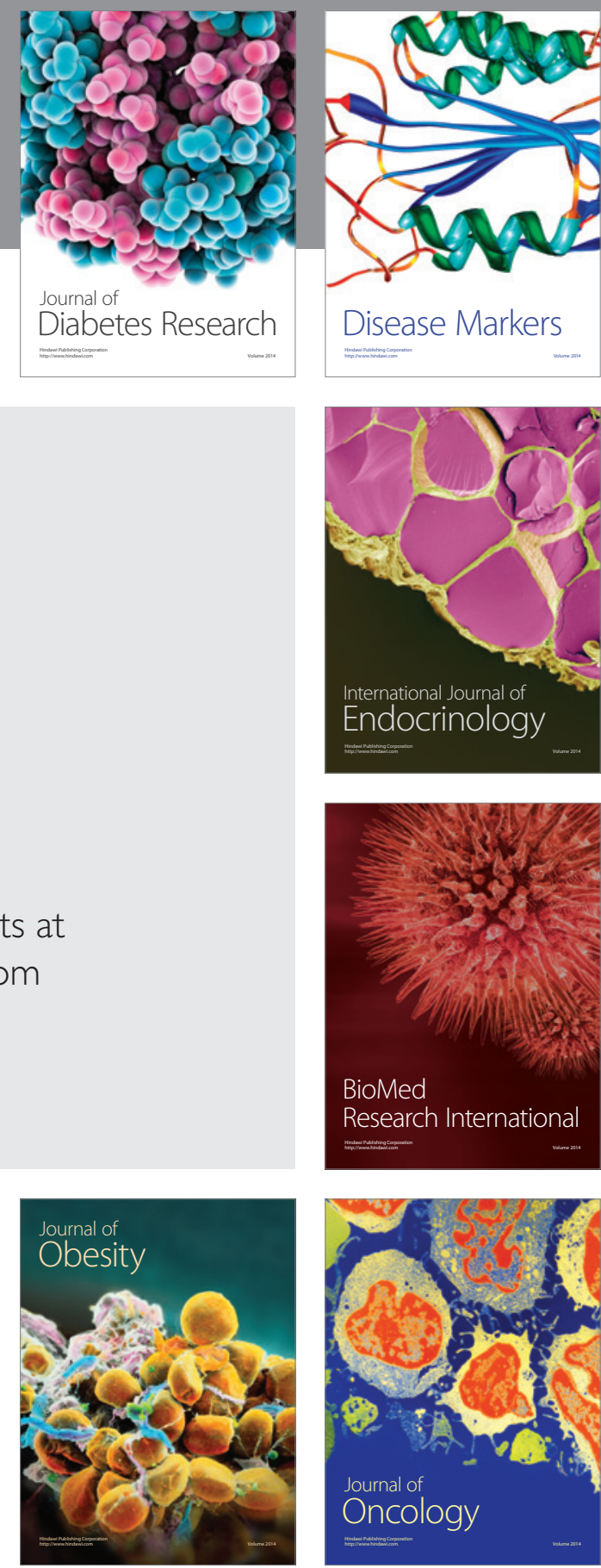

Disease Markers
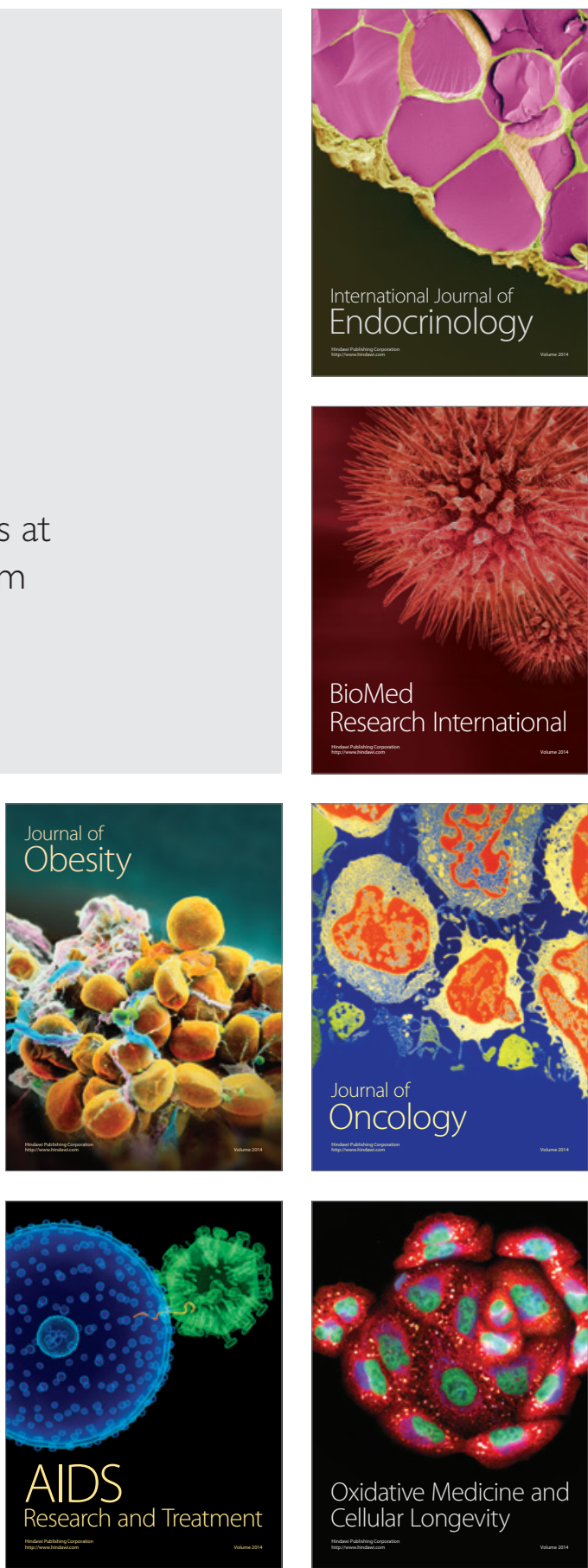